\title{
Mapping the Hidden Universe: The Galaxy Distribution in the Zone of Avoidance
}

\author{
Renée C. Kraan-Korteweg ${ }^{1}$ and Sebastian Juraszek ${ }^{2,3}$ \\ ${ }^{1}$ Departamento de Astronomía, Universidad de Guanajuato, Apartado Postal 144, \\ Guanajuato GTO 36000, Mexico \\ kraan@astro.ugto.mx \\ ${ }^{2}$ School of Physics, University of Sydney, NSW 2006, Australia \\ ${ }^{3}$ ATNF, CSIRO, PO Box 76, Epping, NSW 2121, Australia \\ sjurasze@atnf.csiro.au \\ Received 1999 August 26, accepted 1999 October 26
}

\begin{abstract}
Due to the foreground extinction of the Milky Way, galaxies become increasingly faint as they approach the Galactic Equator creating a 'zone of avoidance' (ZOA) in the distribution of optically visible galaxies of about $25 \%$. A 'whole-sky' map of galaxies is essential, however, for understanding the dynamics in our local Universe, in particular the peculiar velocity of the Local Group with respect to the Cosmic Microwave Background and velocity flow fields such as in the Great Attractor (GA) region. The current status of deep optical galaxy searches behind the Milky Way and their completeness as a function of foreground extinction will be reviewed. It has been shown that these surveys - which in the mean time cover the whole ZOA (Figure 2)-result in a considerable reduction of the ZOA from extinction levels of $\mathrm{A}_{\mathrm{B}}=1^{\mathrm{m}} .0$ (Figure 1) to $\mathrm{A}_{\mathrm{B}}=3^{\mathrm{m}} .0$ (Figure 3 ). In the remaining, optically opaque $\mathrm{ZOA}$, systematic $\mathrm{Hi}$ surveys are powerful in uncovering galaxies, as is demonstrated for the GA region with data from the full sensitivity Parkes Multibeam Hi survey $\left(300^{\circ} \leq \ell \leq 332^{\circ},|b| \leq 5.5\right.$, Figure 4$)$.
\end{abstract}

Keywords: zone of avoidance — surveys — ISM: dust, extinction — large-scale structure of universe

\section{Introduction}

In 1988, Lynden-Bell \& Lahav for the first time prepared a 'whole-sky' distribution of the extragalactic light to map the density enhancements in the local Universe, to compare them with cosmic flow fields and to determine the gravity field on the Local Group. Assuming that light traces mass, this could be realised through a diameter-coded distribution of galaxies larger than $\mathrm{D} \gtrsim 1^{\prime} .0$ taken from the following galaxy catalogs: the Uppsala General Catalog UGC (Nilson 1973) for the north $(\delta \geq-2 \circ 5)$, the ESO Uppsala Catalog (Lauberts $1982)$ for the south $\left(\delta \leq-17^{\circ} .5\right)$, while the missing strip $(-17.5<\delta<-2.5)$ was filled with data from the Morphological Catalog of Galaxies MCG (Vorontsov-Velyaminov \& Archipova 1963-74).

Because these optical galaxy catalogs are limited to the largest galaxies they become increasingly incomplete close to the Galactic equator where the dust thickens, reducing the apparent diameters of the galaxies. Added to this are the growing number of foreground stars which fully or partially block the view of galaxy images. This is clearly demonstrated in Figure 1, where such a light distribution is presented in an Aitoff equal-area projection centred on the Galactic plane. The same corrections as advocated in Lahav (1987) have been applied to homogenise the data of the three different galaxy catalogs, i.e. $\mathrm{D}_{25}=1 \cdot 15 \cdot \mathrm{D}_{\mathrm{UGC}}, \mathrm{D}_{25}=0 \cdot 96 \cdot \mathrm{D}_{\mathrm{ESO}}$ and $\mathrm{D}_{25}=1 \cdot 29 \cdot \mathrm{D}_{\mathrm{MCG}}$. A cut-off at $\mathrm{D}=1.3$ was imposed - the completeness limit of the respective catalogs according to Hudson \& Lynden-Bell (1991).

Figure 1 clearly displays the irregularity in the distribution of galaxies in the nearby Universe with its dynamically important density enhancements such as the Local Supercluster visible as a circle (the Supergalactic Plane) centred on the Virgo cluster at $\ell=284^{\circ}, b=74^{\circ}$, the Perseus-Pisces chain bending into the $\mathrm{ZOA}$ at $\ell=95^{\circ}$ and $\ell=165^{\circ}$, the general overdensity in the Cosmic Microwave Background dipole direction $\left(\ell=280^{\circ}, b=27^{\circ}\right.$, Kogut et al. 1993) and the Great Attractor region centred on $\ell=320^{\circ}, b=0^{\circ}$ (Kolatt, Dekel \& Lahav 1995) with the Hydra $\left(270^{\circ}, 27^{\circ}\right)$, Antlia $\left(273^{\circ}, 19^{\circ}\right)$, Centaurus $\left(302^{\circ}, 22^{\circ}\right)$ and Pavo $\left(332^{\circ},-24^{\circ}\right)$ clusters.

Most conspicuous in this distribution is, however, the very broad, nearly empty band of about $20^{\circ}$ : the Zone of Avoidance. Comparing this band with the $100 \mu \mathrm{m}$ dust extinction maps of the DIRBE 


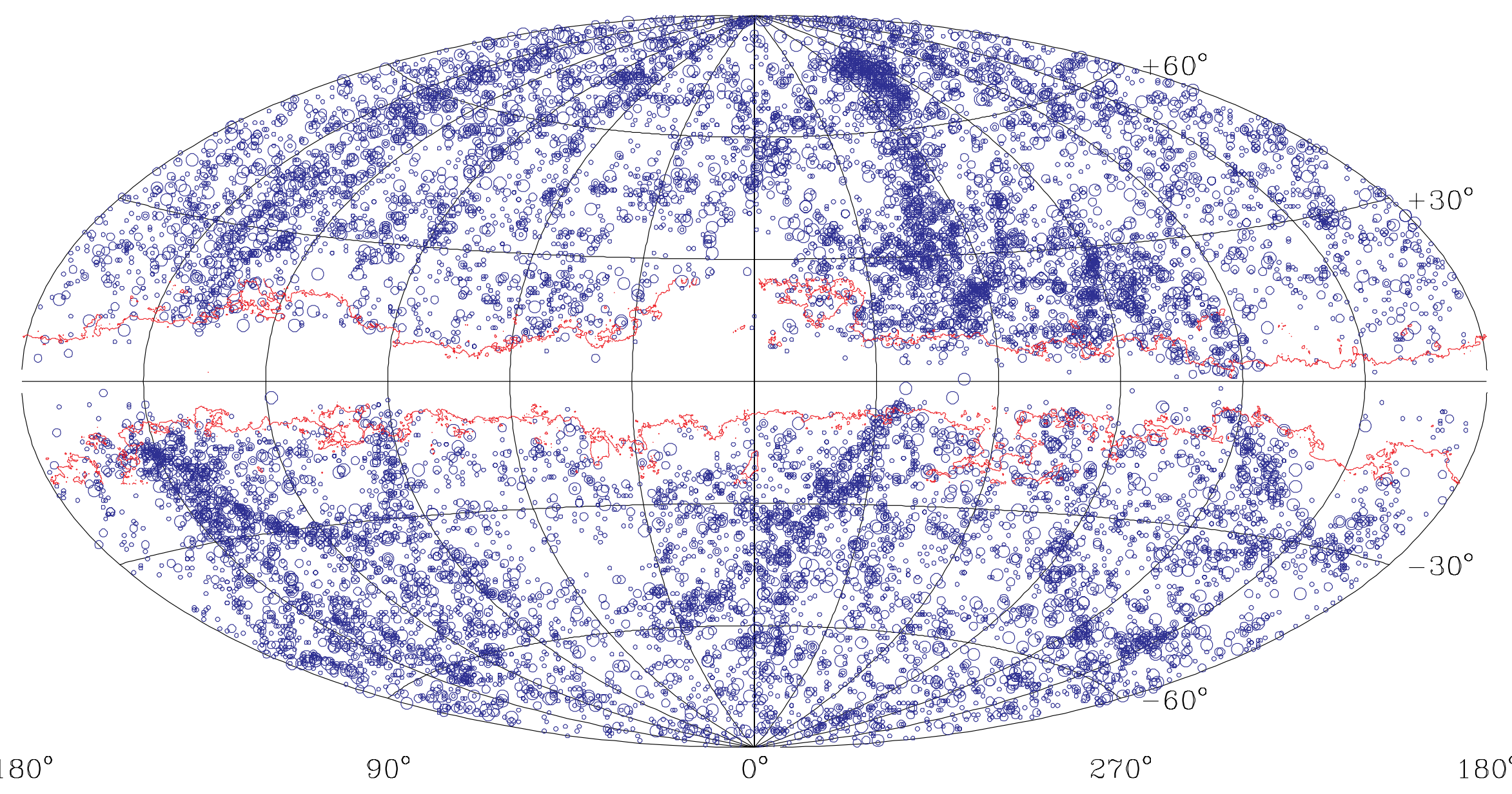

Figure 1-Aitoff equal-area projection in Galactic coordinates of galaxies with $\mathrm{D} \geq 1^{\prime} 3$. The galaxies are diameter-coded: small circles represent galaxies with $1^{\prime} 3 \leq \mathrm{D}<2^{\prime}$, medium circles $2^{\prime} \leq \mathrm{D}<3^{\prime}$, and large circles $\mathrm{D} \geq 3^{\prime}$. The contour marks absorption in the blue of $\mathrm{A}_{\mathrm{B}}=1^{\mathrm{m}} 0$ as determined from the dust extinction maps of Schlegel, Finkbeiner \& Davis (1998). The displayed contour surrounds the area where the galaxy distribution becomes incomplete (the ZOA) remarkably well. 


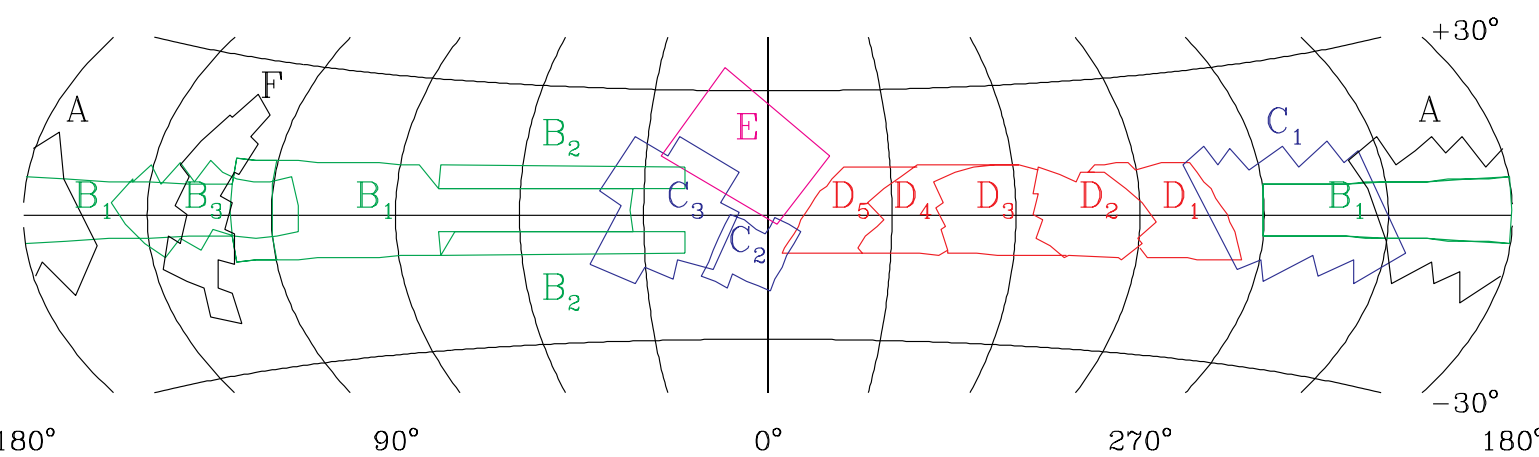

Figure 2-An overview of the different optical galaxy surveys in the ZOA centred on the Galaxy. The labels identifying the search areas are explained in the text. Note that the surveyed regions entirely cover the ZOA defined by the foreground extinction level of $\mathrm{A}_{\mathrm{B}}=1^{\mathrm{m}} .0$ in Figure 1 .

experiment (Schlegel, Finkbeiner \& Davis 1998) we found that the $\mathrm{ZOA}$ - the area where our galaxy counts are severely incomplete - is described almost perfectly by the extinction contour $\mathrm{A}_{\mathrm{B}}=1$. $\mathrm{m} 0$ [where $\mathrm{A}_{\mathrm{B}}=4 \cdot 14 \cdot \mathrm{E}(\mathrm{B}-\mathrm{V})$, Cardelli, Clayton \& Mathis $1989]$.

\section{Deep Optical Galaxy Searches}

Systematic deeper searches for 'partially obscured' galaxies - down to fainter magnitudes and smaller dimensions $\left(\mathrm{D} \leq 0^{\prime}{ }^{\prime} 1\right)$ than existing catalogs - have been performed with the aim to reduce the ZOA. Using existing sky surveys (POSS I and POSS II in the north and the ESO/SERC surveys in the south), the whole ZOA has in the mean time been visually surveyed for galaxies. Here, examination by eye is still the best technique. A separation of galaxy and star images can as yet not be done by automated measuring machines (e.g. COSMOS or APM) on a viable basis below $|b| \lesssim 10^{\circ}-15^{\circ}$, though surveys by eye are clearly both very trying and time consuming, and possibly not as objective.

The various surveyed regions are displayed in Figure 2. Details and results on the uncovered galaxy distributions for the various regions are discussed in:

A: the Perseus-Pisces Supercluster by Pantoja et al. (1997);

$\mathrm{B}_{1-3}$ : the northern Milky Way $\left[\mathrm{B}_{1}\right.$ by Seeberger et al. (1994), Seeberger, Saurer \& Weinberger (1996), Lercher, Kerber \& Weinberger (1996), Saurer, Seeberger \& Weinberger (1997) and Seeberger \& Saurer 1998 from POSS I; $B_{2}$ by Marchiotti, Wildauer \& Weinberger (1999) from POSS II; B 3 by Weinberger, Gajdosik \& Zanin (1999) from POSS II];

$\mathrm{C}_{1-3}$ : the Puppis region by Saito et al. (1990, 1991) $\left[\mathrm{C}_{1}\right]$, the Sagittarius/Galactic region by Roman, Nakanishi \& Saito (1998) $\left[\mathrm{C}_{2}\right]$, and the Aquila and Sagittarius region by Roman et al. (1996) $\left[\mathrm{C}_{3}\right]$;

$\mathrm{D}_{1-5}$ : the southern Milky Way [the Hydra to Puppis region $\left[D_{1}\right]$ by Salem \& Kraan-Korteweg
(2000), the Hydra/Antlia Supercluster region $\left[\mathrm{D}_{2}\right]$ by Kraan-Korteweg (2000), the Crux region $\left[\mathrm{D}_{3}\right]$ by Woudt (1998) and Woudt \& Kraan-Korteweg (2000a), the GA region $\left[\mathrm{D}_{4}\right]$ by Woudt (1998) and Woudt \& Kraan-Korteweg (2000b), and the Scorpius region $\left[\mathrm{D}_{5}\right]$ by Fairall \& Kraan-Korteweg (2000)];

E: the Ophiuchus Supercluster by Wakamatsu et al. (1994) and Hasegawa et al. (1999);

F: the northern GP/SGP crossing by Hau et al. (1996).

These surveys have uncovered over 50,000 previously unknown galaxies and are not biased with respect to any particular morphological type. Although the various optical surveys are based on different plate material and were performed by different groups, the search techniques overall are similar. All $\mathrm{ZOA}$ regions have been searched to diameter limits of at least $\mathrm{D} \gtrsim 0.2$. This is considerably deeper than the previously regarded 'whole-sky' catalogs with their completeness limits of $\mathrm{D} \gtrsim 1$ '.3. How can these catalogs be merged to arrive at a well-defined whole-sky galaxy distribution with a reduced ZOA?

\section{Completeness of Optical Galaxy Searches}

In order to merge the various deep optical ZOA surveys with existing galaxy catalogs, Kraan-Korteweg (2000) and Woudt (1998) have analysed the completeness of their ZOA galaxy catalogs - the Hy/Ant $\left[\mathrm{D}_{2}\right]$, Crux $\left[\mathrm{D}_{3}\right]$ and $\mathrm{GA}\left[\mathrm{D}_{4}\right]$ region-as a function of the foreground extinction.

By studying the apparent diameter distribution as a function of the extinction $\mathrm{E}(\mathrm{B}-\mathrm{V})$ (Schlegel, Finkbeiner \& Davis 1998) as well as the location of the flattening in the slope of the cumulative diameter curves $\log \mathrm{D}-\log \mathrm{N}$ for various extinction intervals (cf. Figures 5 and 6 in Kraan-Korteweg 1999), we conclude that our optical ZOA surveys are complete to an apparent diameter of $\mathrm{D}=14^{\prime \prime}$ where the diameters correspond to an isophote of $24.5 \mathrm{mag} / \operatorname{arcsec}^{2}$ (Kraan-Korteweg 2000)-for extinction levels less than $A_{B}=3^{m}$. 0 . 
Figure 3-Aitoff equal-area distribution of ESO, UGC, MCG galaxies with extinction-corrected diameters $\mathrm{D}^{\circ} \geq 1^{\prime} 3$, including galaxies identified in the optical ZOA galaxy searches for extinction-levels of $A_{B} \leq 3^{\mathrm{m}} 0$ (contour). The diameters are coded as in Figure 1 . With the exception of the areas for which either the positions of the galaxies or their diameters are not yet available (demarcated areas), the ZOA could be reduced considerably compared to Figure 1. 
How about the intrinsic diameters, i.e. the diameters galaxies would have if they were unobscured? A spiral galaxy seen through an extinction of $\mathrm{A}_{\mathrm{B}}=1^{\mathrm{m}}$. 0 will, for example, be reduced to $\sim 80 \%$ of its unobscured size. Only $\sim 22 \%$ of a (spiral) galaxy's original dimension is seen when it is observed through $\mathrm{A}_{\mathrm{B}}=3^{\mathrm{m}} .0$. In 1990, Cameron derived analytical descriptions to correct for the obscuration effects by artificially absorbing the intensity profiles of unobscured galaxies. These corrections depend quite strongly on morphological type due to the difference in surface brightness profiles and mean surface brightness between early-type galaxies and spiral galaxies. Applying these corrections, we find that at $\mathrm{A}_{\mathrm{B}}=3^{\mathrm{m}} .0$, an obscured spiral or elliptical galaxy at our apparent completeness limit of $\mathrm{D}=14^{\prime \prime}$ would have an intrinsic diameter of $\mathrm{D}^{\circ} \approx 60^{\prime \prime}$ or $\mathrm{D}^{\circ} \approx 50^{\prime \prime}$, respectively. At extinction levels higher than $\mathrm{A}_{\mathrm{B}}=3^{\mathrm{m}} \cdot 0$, an elliptical galaxy with $\mathrm{D}^{\circ} \approx 60^{\prime \prime}$ would appear smaller than the completeness limit $\mathrm{D}=14^{\prime \prime}$ and might have gone unnoticed. The optical galaxy catalog discussed here should therefore be complete to $\mathrm{D}^{\circ} \geq 60^{\prime \prime}$ for galaxies of all morphological types down to extinction levels of $A_{B} \leq 3^{m}$. 0 , with the possible exception of extremely low-surface brightness galaxies. Only intrinsically very large and bright galaxies - particularly galaxies with high surface brightness - will be recovered in deeper extinction layers. This completeness limit could be confirmed by independently analysing the diameter versus extinction and the cumulative diameter diagrams for extinction-corrected diameters.

We can thus supplement the ESO, UGC and MCG catalogs - which are complete to $\mathrm{D}=1.3$ with galaxies from optical ZOA galaxy searches that have $\mathrm{D}^{\circ} \geq 1^{\prime} \cdot 3$ and $\mathrm{A}_{\mathrm{B}} \leq 3^{\mathrm{m}}$. 0 . As our completeness limit lies well above the ESO, UGC and MCG catalogs, we can assume that the other similarly performed optical galaxy searches in the ZOA should also be complete to $\mathrm{D}^{\circ}=1.3$ for extinction levels of $\mathrm{A}_{\mathrm{B}} \leq 3^{\mathrm{m}} \cdot 0$.

In Figure 3 we have then taken the first step in arriving at an improved whole-sky galaxy distribution with a reduced ZOA. In this Aitoff projection we have plotted all the UGC, ESO, MCG galaxies that have extinction-corrected diameters $\mathrm{D}^{\circ} \geq 1.3$ (remember that galaxies adjacent to the optical galaxy search regions are also affected by absorption though to a lesser extent: $A_{B} \leq 1^{\mathrm{m}} \cdot 0$ ), and added the galaxies from the various optical surveys with $\mathrm{D}^{\circ}=1.3$ and $A_{B} \leq 3^{m} .0$ for which positions and diameters were available. The regions for which these data are not yet available are marked in Figure 3. As some searches were performed on older generation POSS I plates, which are less deep compared to the second generation POSS II and ESO/SERC plates, an additional correction was applied to those diameters, i.e. the same correction as for the UGC galaxies which also are based on POSS I survey material $\left(\mathrm{D}_{25}=1 \cdot 15 \cdot \mathrm{D}_{\mathrm{POSSI}}\right)$.

A comparison of Figure 1 with Figure 3 demonstrates convincingly how the deep optical galaxy searches realise a considerable reduction of the $\mathrm{ZOA}$; we can now trace the large-scale structures in the nearby Universe to extinction levels of $A_{B}=3^{m} .0$. Inspection of Figure 3 reveals that the galaxy density enhancement in the GA region is even more pronounced and a connection of the Perseus-Pisces chain across the Milky Way at $\ell=165^{\circ}$ more likely. Hence, these supplemented whole-sky maps certainly should improve our understanding of the velocity flow fields and the total gravitational attraction on the Local Group.

Redshift follow-ups of well-defined samples of ZOA galaxies will be important in analysing the large-scale structures in redshift space. Systematic redshift surveys have been performed for various ZOA regions and revealed a number of dynamically important structures such as

- the rich, massive $\left(\sim 2-5 \times 10^{15} \mathrm{M}_{\odot}\right)$ cluster A3627 at $(\ell, b, v) \sim\left(325^{\circ},-7^{\circ}, 4882 \mathrm{~km} \mathrm{~s}^{-1}\right)$ which seems to constitute the previously unrecognised but predicted density peak at the bottom of the potential well of the Great Attractor (KraanKorteweg et al. 1996)

- the $3 \mathrm{C} 129$ cluster at $(\ell, b, v) \sim\left(160^{\circ}, 0^{\circ}, 5500 \mathrm{~km}\right.$ $\left.\mathrm{s}^{-1}\right)$ connecting Perseus-Pisces and A569 across the Galactic Plane (Chamaraux et al. 1990; Pantoja et al. 1997)

- and the Ophiuchus supercluster at $(\ell, b, v) \sim$ $\left(0^{\circ}, 8^{\circ}, 8500 \mathrm{~km} \mathrm{~s}^{-1}\right)$ behind the Galactic Centre (Wakamatsu et al. 1994; Hasegawa et al. 1999).

Optical galaxy searches, however, fail in the most opaque part of the Milky Way, the region encompassed by the $\mathrm{A}_{\mathrm{B}}=3^{\mathrm{m}} \cdot 0$ contour in Figure 3 a sufficiently large region to hide further dynamically important galaxy densities. Here, systematic HI surveys have proven very powerful as the Galaxy is transparent to the $21-\mathrm{cm}$ line radiation of neutral hydrogen and Hi-rich galaxies can readily be found through detection of their redshifted 21-cm emission.

\section{HI Galaxy Searches in the ZOA}

In March 1997, a systematic blind Hi survey began in the most opaque part of the southern Milky Way $\left(213^{\circ} \leq \ell \leq 33^{\circ} ;|b| \leq 5.5\right)$ with the Multibeam (MB) receiver (13 beams in the focal plane array) at the $64 \mathrm{~m}$ Parkes telescope. The ZOA is being surveyed along constant Galactic latitudes in 23 contiguous fields of length $\Delta \ell=8^{\circ}$. The ultimate goal is 25 scans per field where adjacent strips will be offset in latitude by $\Delta b=1^{\prime} .5$ for homogeneous sampling. With an effective integration time of $25 \mathrm{~min} /$ beam we obtain a $3 \sigma$ detection limit of $25 \mathrm{mJy}$. The survey covers a velocity range of 

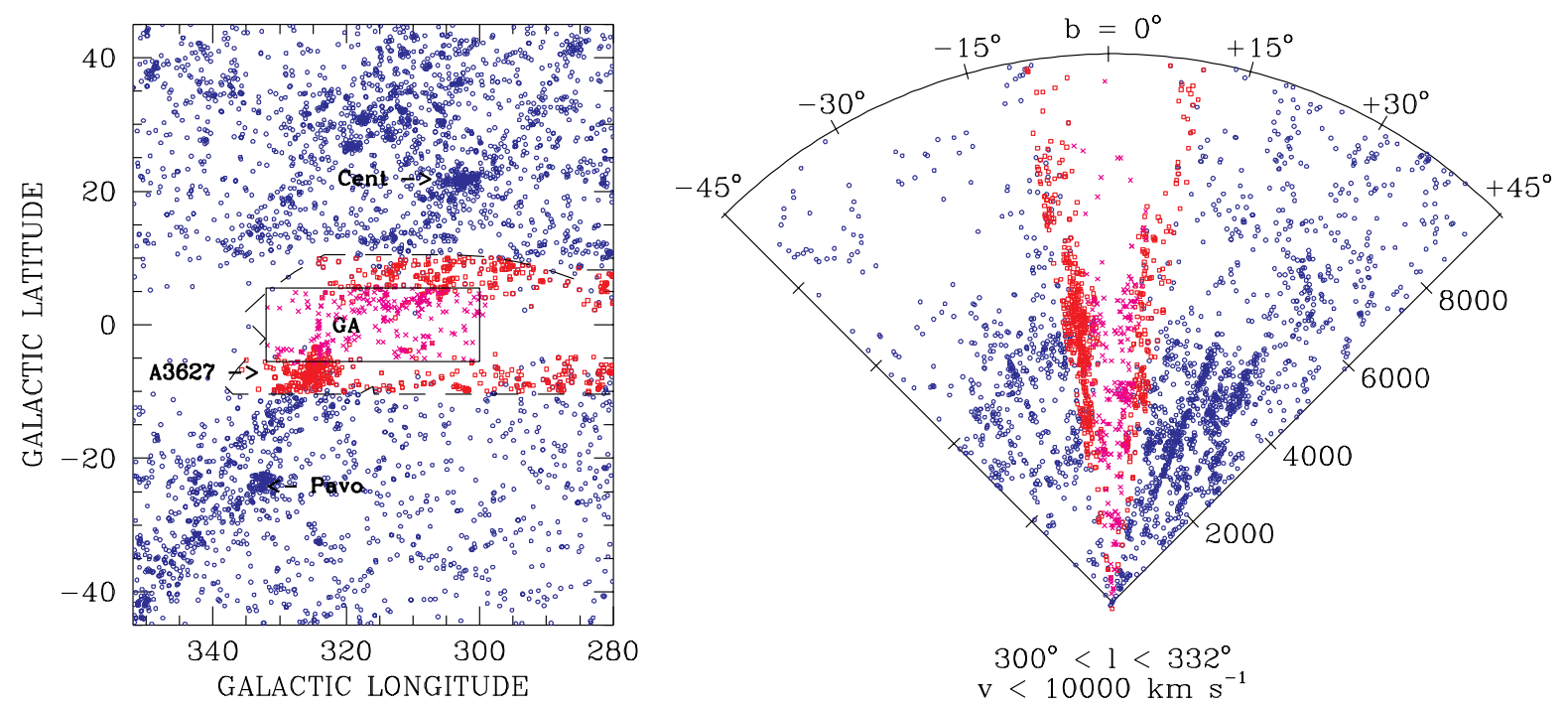

Figure 4-A sky distribution (left) and redshift cone (right) for galaxies with $v<10000 \mathrm{~km} \mathrm{~s}^{-1}$ in the GA region. Circles mark redshifts from the literature (LEDA), squares mark redshifts from the optical galaxy search in the Hy/Ant-Crux-GA regions (outlined on left panel), and crosses mark detections in the full-sensitivity Hi MB-ZOA survey (box).

$-1200 \lesssim v \lesssim 12700 \mathrm{~km} \mathrm{~s}^{-1}$, with a channel spacing of $13 \cdot 2 \mathrm{~km} \mathrm{~s}^{-1}$ per channel, and will be sensitive to normal spiral galaxies well beyond the Great Attractor region.

So far, a shallow survey based on two of the foreseen 25 driftscan passages has been analysed (cf. Kraan-Korteweg, Koribalski \& Juraszek 1998; Henning et al. 1999). A total of 107 galaxies were catalogued with peak Hi flux densities of $\gtrsim 80 \mathrm{mJy}$ (rms $=15$ mJy after Hanning smoothing) and their detection shows no dependence on Galactic latitude, nor the amount of foreground obscuration through which they have been detected.

Four cubes centred on the Great Attractor region $\left(300^{\circ} \geq \ell \geq 332^{\circ},|b| \leq 5.5\right)$ of the full-sensitivity survey have been analysed (Juraszek et al. 2000) and uncovered 236 galaxies above the $3 \sigma$ detection level of $25 \mathrm{mJy} .70 \%$ of the detections had no previous identification.

In the left panel of Figure 4, a sky distribution centred on the GA region displays all galaxies with redshifts $v \leq 10000 \mathrm{~km} \mathrm{~s}^{-1}$. Next to redshifts from the literature (circles; LEDA), redshifts from the follow-up observations of Kraan-Korteweg and collaborators in the Hy/Ant-Crux-GA ZOA surveys (dashed area) are plotted. They clearly reveal the prominence of the cluster A3627 at $(\ell, b, v)=$ $\left(325^{\circ}, \quad-7^{\circ}, 4882 \mathrm{~km} \mathrm{~s}^{-1}\right.$, Kraan-Korteweg et al. 1996) close to the core of the GA region at $(\ell, b, v)=\left(320^{\circ}, 0^{\circ}, 4500 \mathrm{~km} \mathrm{~s}^{-1}\right)$ as predicted by Kolatt, Dekel \& Lahav (1995). Adding now the new detections from the systematic blind HI MB-ZOA survey (box), for the first time we can trace structures all the way across the Milky Way. The new picture seems to suggest that the GA overdensity is a 'great-wall' like structure starting at the Pavo cluster, having its core at the A3627 cluster and then bending over towards shorter longitudes across the ZOA.

This becomes even clearer in the right panel of Figure 4 where the galaxies are displayed in a redshift cone out to $v \leq 10000 \mathrm{~km} \mathrm{~s}^{-1}$ for the longitude range $300^{\circ} \leq \ell \leq 332^{\circ}$ analysed so far of the $\mathrm{MB}$ full-sensitivity data. The A3627 cluster is clearly the most massive galaxy cluster uncovered by the various surveys in the GA region and therefore the most likely candidate for the previously unidentified, but predicted, density peak at the bottom of the potential well of the GA overdensity.

Finding a hitherto uncharted further cluster of galaxies at the heart of the GA would have serious implications for our current understanding of this massive overdensity in the local Universe. Various indications suggest that PKS1343-601, the second brightest extragalactic radio source in the southern sky $\left(f_{20 \mathrm{~cm}}=79 \mathrm{Jy}\right.$, McAdam 1991), might form the centre of yet another highly obscured rich cluster, particularly as it also shows significant Xray emission (cf. Kraan-Korteweg \& Woudt 1999 for further details). At $(\ell, b) \sim\left(310^{\circ}, 2^{\circ}\right)$, this radio galaxy lies behind an obscuration layer of about 12 magnitudes of extinction in the B-band; hence optical surveys are ineffective. Still, West \& Tarenghi (1989) observed this source and identified it - with an extinction-corrected diameter of $\mathrm{D}^{\circ} \approx 4^{\prime}$ and a recession velocity of $v=3872 \mathrm{~km} \mathrm{~s}^{-1}$ — as a giant elliptical galaxy. Giant ellipticals generally reside at the cores of clusters.

Interestingly enough, the $\mathrm{HI}$ MB survey does uncover a significant excess of galaxies at this 
position in velocity space (cf. Figure 4). However, we do not see a 'finger of God', the characteristic signature of a cluster in redshift space. Could it be that too many central cluster galaxies are missed by the Hi observations because spiral galaxies generally avoid the cores of clusters? The existence of this possible cluster still remains a mystery. Meanwhile, this prospective cluster has been imaged in the near infrared (Woudt et al. in progress), where extinction effects are less severe compared to the optical, and which should uncover early-type cluster members if they are there. The forthcoming results should then unambiguously settle the question of whether another cluster forms part of the GA supercluster.

\section{Acknowledgments}

The collaboration with our colleagues in the optical surveys and redshift follow-up observations is greatly appreciated: C. Balkowski, V. Cayatte, A. P. Fairall, C. Salem, P. A. Woudt, and the HIPASS ZOA team members R. D. Ekers, A. J. Green, R. F. Haynes, P. A. Henning, M. J. Kesteven, B. Koribalski, R. M. Price, E. Sadler and L. Staveley-Smith.

\section{References}

Cameron, L. M. 1990, A\&A, 233, 16

Cardelli, J. A., Clayton, G. C., \& Mathis, J. S. 1989, ApJ, 345,245

Chamaraux, P., Cayatte, V., Balkowski, C., \& Fontanelli, P. 1990, A\&A, 229, 340

Fairall, A. P., \& Kraan-Korteweg, R. C. 2000, in preparation $\left[D_{5}\right]$

Hasegawa, T., Wakamatsu, K., Malkan, M., et al. 1999, MNRAS, in press $[\mathrm{E}]$

Hau, G. K. T., Ferguson, H. C., Lahav, O., et al. 1996, MNRAS, 277, $125[\mathrm{~F}]$

Henning, P. A., Staveley-Smith, L., Kraan-Korteweg, R. C., \& Sadler, E. M. 1999, PASA, 16, 35

Hudson, M., \& Lynden-Bell, D. 1991, MNRAS, 252, 219

Juraszek, S., Staveley-Smith, L., Kraan-Korteweg, R. C., Green, A. J., Ekers, R. D., Henning, P. A., Koribalski, B. S., Sadler, E. M., \& Schröder, A. C. 2000, in preparation

Kogut, A., Lineweaver, C., Smoot, G. F., et al. 1993, ApJ, 419,1

Kolatt, T., Dekel, A., \& Lahav, O. 1995, MNRAS, 275, 797

Kraan-Korteweg, R. C. 2000, A\&ASS, 141, 123

Kraan-Korteweg, R. C., Koribalski, B., \& Juraszek, S. 1998, in Looking Deep in the Southern Sky, ed. R. Morganti \& W. Couch (New York: Springer), p. 23
Kraan-Korteweg, R. C., \& Woudt, P. A. 1999, PASA, 16, 53

Kraan-Korteweg, R. C., Woudt, P. A., Cayatte, V., et al. 1996, Nature, 379, 519

Lahav, O. 1987, MNRAS, 225, 213

Lauberts, A. 1982, The ESO/Uppsala Survey of the ESO (B) Atlas (Garching: ESO)

Lercher, G., Kerber, F., \& Weinberger, R. 1996, A\&ASS, $117,369,\left[\mathrm{~B}_{1}\right]$

Lynden-Bell, D., \& Lahav, O. 1988, in Large-Scale Motions in the Universe, ed. V. C. Rubin \& G. V. Coyne (Princeton Univ. Press), p. 199

McAdam, W. B. 1991, PASA, 9, 255

Marchiotti, W., Wildauer, H., \& Weinberger, R. 1999, in progress $\left[\mathrm{B}_{2}\right]$

Nilson, P. 1973, Uppsala General Catalog of Galaxies (University of Uppsala)

Pantoja, C. A., Altschuler, D. R., Giovanardi, C., \& Giovanelli, R. 1997, AJ, 113, 905 [A]

Roman, A. T., Nakanishi, K., Tomita, A., \& Saito, M. 1996, PASJ, 48, $679\left[\mathrm{C}_{3}\right]$

Roman, A. T., Nakanishi, K., \& Saito, M. 1998, PASJ, 50, $37\left[\mathrm{C}_{2}\right]$

Saito, M., Ohtani, A., Asomuna, A., et al. 1990, PASJ, 42, $603\left[\mathrm{C}_{1}\right]$

Saito, M., Ohtani, A., Baba, A., et al. 1991, PASJ, 43, 449 $\left[\mathrm{C}_{1}\right]$

Salem, C., \& Kraan-Korteweg, R. C. 2000, in preparation $\left[\mathrm{D}_{1}\right]$

Saurer, W., Seeberger, R., \& Weinberger, R. 1997, A\&ASS, $126,247,\left[\mathrm{~B}_{1}\right]$

Schlegel, D. J., Finkbeiner, D. P., \& Davis, M. 1998, ApJ, 500,525

Seeberger, R., \& Saurer, W. 1998, A\&ASS, 127, 101 [B 1 ]

Seeberger, R., Saurer, W., Weinberger, R., \& Lercher, G. 1994, in Unveiling Large-Scale Structures behind the Milky Way, ed. C. Balkowski \& R. C. Kraan-Korteweg, ASP Conf. Ser. 67 (San Francisco), p. $81\left[\mathrm{~B}_{1}\right]$

Seeberger, R., Saurer, W., \& Weinberger, R. 1996, A\&ASS, $117,1\left[\mathrm{~B}_{1}\right]$

Vorontsov-Velyaminov, B., \& Archipova, V. 1963-74, Morphological Catalog of Galaxies, Parts 2-5 (Moscow: Moscow University)

Wakamatsu, K., Hasegawa, T., Karoji, H., et al. 1994, in Unveiling Large-Scale Structures behind the Milky Way, ed. C. Balkowski \& R. C. Kraan-Korteweg, ASP Conf. Ser. 67 (San Francisco), p. 131 [E]

Weinberger, R., Gajdosik, M., \& Zanin, C. 1999, A\&ASS, 137, $293\left[\mathrm{~B}_{5}\right]$

West, R. M., \& Tarenghi, M. 1989, A\&A, 223, 61

Woudt, P. A. 1998, PhD thesis, University of Cape Town $\left[\mathrm{D}_{3}, \mathrm{D}_{4}\right]$

Woudt, P. A., \& Kraan-Korteweg, R. C. 2000a, A\&ASS, in preparation $\left[\mathrm{D}_{3}\right]$

Woudt, P. A., \& Kraan-Korteweg, R. C. 2000b, A\&ASS, in preparation $\left[\mathrm{D}_{4}\right]$ 